\title{
Mortar DG Method with Staggered Hybridization for Rayleigh Waves Simulation
}

\author{
Jie $\mathrm{Du}^{1}$ and Eric Chung ${ }^{2, *}$ \\ ${ }^{1}$ Yau Mathematical Sciences Center, Tsinghua University, Beijing 100084, P.R. China. \\ 2 Department of Mathematics, The Chinese University of Hong Kong, Shatin, \\ New Territories, Hong Kong SAR, China.
}

Received 19 February 2020; Accepted (in revised version) 27 April 2020

\begin{abstract}
The simulation of Rayleigh waves is important in a variety of geophysical applications. The computational challenge is the fact that very fine mesh is necessary as the waves are concentrated at the free surface and decay exponentially away from the free surface. To overcome this challenge and to develop a robust high order scheme for the simulation of Rayleigh waves, we develop a mortar discontinuous Galerkin method with staggered hybridization. The use of the mortar technique allows one to use fine mesh in only a local region near the free surface, and use coarse mesh in most of the domain. This approach reduces the computational cost significantly. The staggered hybridization allows the preservation of the strong symmetry of the stress tensor without complicated construction of basis functions. In particular, the basis functions are piecewise polynomial without any continuity requirement, and the coupling of the basis functions is performed by using carefully chosen hybridized variables. The resulting scheme is explicit in time, and only local saddle point system are solved for each time step. We will present several benchmark problems to demonstrate the performance of the proposed method.
\end{abstract}

AMS subject classifications: 65M32, 65M60

Key words: Discontinuous Galerkin method, elastic wave equations, Rayleigh wave, mortar formulation, hybridization.

\section{Introduction}

Accurate elastodynamic simulations are of critical importance in a variety of geophysical applications. The staggered grid finite difference methods [21,23,29] is a class of efficient numerical schemes for accurate elastic wave computations, and they are widely used in

*Corresponding author. Email addresses: jdu@tsinghua.edu.cn (J. Du), tschung@math.cuhk.edu.hk (E. Chung) 
a number of applications. For computational domains with irregular geometries, such as non-flat topography, the accuracy of these methods diminishes. For many realistic applications, one needs accurate and efficient computational techniques that can be applied to domains with complex geometries or non-flat interfaces. The discontinuous Galerkin (DG) methods can be used to tackle these computational challenges. The DG method approximates the solution by using piecewise polynomial functions defined on unstructured meshes, which can triangulate complex domain geometries. For example, various DG methods are proposed in $[1,4,13-16,18,19,22,24,25,27,28,30]$.

The use of staggered mesh for computational wave propagation has shown its prominence in applications. Motivated by the staggered grid finite difference schemes, the staggered discontinuous Galerkin (SDG) method is developed with the goal of achieving high order accuracy on domains with irregular geometries and keeping the advantages of using staggered meshes. The SDG methods are successfully applied to both the acoustic wave equations $[7,8,20]$ and the elastic wave equations $[6,9]$ as well as other applications $[3,17,31]$. The use of staggered mesh in discontinuous Galerkin method offers several additional advantages such as energy conservation, optimal rate of convergence and low dispersion error $[2,9]$. Another key feature of the proposed method in this paper is the use of staggered hybridization [6]. This staggered hybridization techniques allows one to define the polynomial basis functions locally on each cell in the mesh without enforcing any continuity condition. The coupling of the basis functions is defined by using suitable staggered hybridized variables. One advantage of this technique is that the symmetry of the stress tensor can be enforced strongly on irregular meshes. We remark that the idea of hybridization has been used successfully in discontinuous Galerkin methods $[5,10-12,25,26]$. The technique of staggered hybridization shares many of the advantages of hybridization, such as superconvergence, and gives additional advantages for elastic wave simulations as mentioned above. Furthermore, the proposed method gives explicit time-stepping scheme. In particular, one needs only to solve local saddle point system for each time step. So, the time-stepping is very efficient.

The focus of this paper is efficient simulations of Rayleigh waves. Accurate and efficient computations of Rayleigh waves has important applications in geophysics. From the computational point of view, the simulation of Rayleigh waves is difficult in the sense that a very fine mesh is necessary as the wave is concentrated only on the free surface and decays exponentially in the direction away from the free surface. We also remark that a fine mesh in the whole computational domain is needed even though the wave is only concentrated near the surface. To overcome this computational challenge, we propose the use of mortar technique together with our discontinuous Galerkin method using staggered hybridization. The main idea is to use a fine computational mesh near the surface of the domain, and use a coarse mesh in the rest of the domain. The region of fine mesh is a thin layer near the surface. Thus, the overall degrees of freedom in the whole domain is much reduced. In order to couple the unknown at the interface of the fine and the coarse meshes, we apply the mortar technique. More precisely, we define an additional mortar variable and an additional jump condition to enforce the continuity of 
the elastic displacement. Another important point is to ensure that only local problem is solved in each time step. This can be achieved by a mild assumption on the mesh.

The paper is organized as follows. In Section 2.1, we will present the model equations and notations. Then, in Sections 2.2 and 2.3, we will give the derivation of our discontinuous Galerkin method with mortar formulation. In Section 3, we will present numerical results to demonstrate the performance of our proposed method. We will consider a test for rate of convergence, the standard Lamb's Problem as well as tests with heterogeneous media and curved surfaces. Finally, the paper ends with a conclusion.

\section{Numerical scheme}

\subsection{Model problem and triangulation}

We consider the following first order formulation of elastic wave equations

$$
\begin{aligned}
\rho \frac{\partial \mathbf{u}}{\partial t}-\operatorname{div} \sigma & =\mathbf{f} \\
\mathbf{A} \frac{\partial \sigma}{\partial t}-\varepsilon(\mathbf{u}) & =0
\end{aligned}
$$

in a bounded domain $\Omega \subset \mathbf{R}^{d}(d=2,3)$ with a Lipschitz boundary and within the time interval $[0, T]$. They describe the elastic wave propagation through isotropic media. Here $\mathbf{u}:=\left(u_{1}, \cdots, u_{d}\right)^{T}$ is the velocity field, $\sigma:=\left(\sigma_{i j}\right)$ is the $d \times d$ symmetric stress tensor, and $\mathbf{f}:=\left(f_{1}, \cdots, f_{d}\right)^{T}$ is a given source term. We let $\sigma_{i}$ be the $i$-th row of $\sigma$ and define the divergence as

$$
\operatorname{div} \sigma:=\left(\operatorname{div} \sigma_{1}, \cdots, \operatorname{div} \sigma_{d}\right)^{T} .
$$

Moreover, $\varepsilon(\mathbf{u})=\frac{1}{2}\left(\nabla \mathbf{u}+\nabla \mathbf{u}^{T}\right)$ is a symmetric matrix, where $\nabla \mathbf{u}:=\left(\partial_{j} u_{i}\right)$ is the row-wise gradient of $\mathbf{u}$. The compliance tensor $\mathbf{A}$ can be defined by

$$
\mathbf{A} \sigma:=\frac{1}{2 \mu}\left(\sigma-\frac{\lambda}{2 \mu+d \lambda} \operatorname{tr}(\sigma) I\right)
$$

with the Lamé's parameters $\lambda$ and $\mu$. We assume that the initial data of $\mathbf{u}$ and $\sigma$ are given by

$$
\begin{aligned}
\mathbf{u}(\mathbf{x}, 0)=\mathbf{u}_{0}(\mathbf{x}), & \mathbf{x} \in \Omega, \\
\sigma(\mathbf{x}, 0)=\sigma_{0}(\mathbf{x}), & \mathbf{x} \in \Omega .
\end{aligned}
$$

The boundary condition is assumed as

$$
\begin{aligned}
& \mathbf{u}(\mathbf{x}, t)=\mathbf{u}_{b}(\mathbf{x}, t), \quad \mathbf{x} \in \partial \Omega_{D}, \quad t \in(0, T], \\
& \sigma(\mathbf{x}, t) \mathbf{n}_{e}=\sigma_{b}(\mathbf{x}, t), \quad \mathbf{x} \in \partial \Omega_{N}, \quad t \in(0, T],
\end{aligned}
$$




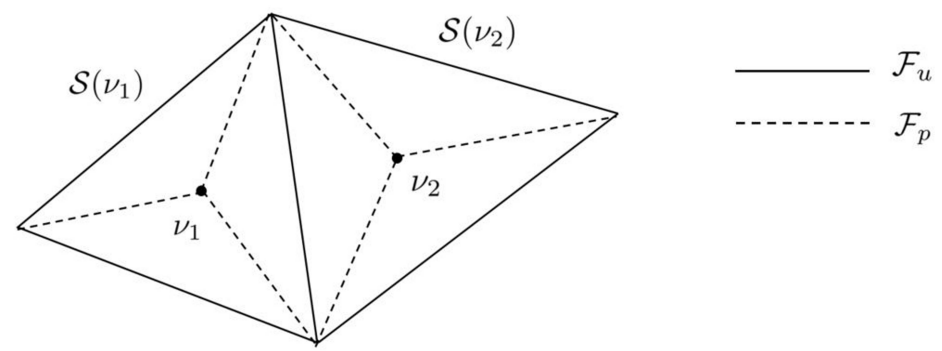

Figure 1: An illustration of the staggered mesh in $\mathbb{R}^{2}$

where $\partial \Omega_{D}$ is the Dirichlet boundary, $\partial \Omega_{N}$ is the Neumann boundary, and $T$ is the terminal time. Here $\mathbf{n}_{e}$ is the outward unit normal vector on the boundary $\partial \Omega$. Also, $\partial \Omega_{D} \cup \partial \Omega_{N}=\partial \Omega$. Our DG method is based on the formulation (2.1)-(2.2), and will compute approximations for $\mathbf{u}, \sigma$.

The computational domain $\Omega$ is divided into a set of $N$ non-overlapping subdomains, $\bar{\Omega}=\bigcup_{i=1}^{N} \overline{\Omega_{i}}$, so that we can use different meshes on different subdomains in order to save the total computational cost. We assume, for simplicity, that $\left\{\Omega_{i}\right\}_{i=1}^{N}$ is a geometrically conforming partition of $\Omega$. We define $\Gamma_{i j}\left(=\partial \Omega_{i} \cap \partial \Omega_{j}\right)$ as the interface shared by the two subdomains $\Omega_{i}$ and $\Omega_{j}$ and denote $\Gamma=\bigcup \Gamma_{i j}$. The main idea is that we use DG method with staggered hybridization on each subdomain and use the mortar technique to couple the unknowns at the interface of different subdomains.

To construct the numerical scheme in each subdomain, every $\Omega_{i}$ is equipped with an initial unstructured triangulation $\mathcal{I} \mathcal{T}_{i}$. The triangulations $\left\{\mathcal{I T}_{i}\right\}_{i=1}^{N}$ can be non-matching across the subdomain interface $\Gamma$. We let $\mathcal{F}_{u, i}$ be the set of all faces in the initial triangulation $\mathcal{I} \mathcal{T}_{i}$ and let $\mathcal{F}_{u, i}^{0} \subset \mathcal{F}_{u, i}$ be the set of all interior faces in $\Omega_{i}$. For each simplex in $\mathcal{I} \mathcal{T}_{i}$, we pick an interior point $v$ and subdivide the simplex into $d+1$ sub-simplices by connecting $v$ to the vertices of the simplex. The union of these $d+1$ sub-simplices is called $\mathcal{S}(v)$. We introduce $\mathcal{N}_{i}$ to denote the set of all such interior points $v$ in $\Omega_{i}$. We denote the set of all the new generated sub-simplices in $\Omega_{i}$ as $\mathcal{T}_{i}$ which is a finer triangulation. We use $\mathcal{F}_{p, i}$ to denote the set of all new faces formed in the subdivision process.

For each element $\tau \in \mathcal{T}_{i}$, we define $\mathbf{n}_{\tau}$ as the outward unit normal vector on $\partial \tau$. We will simply use $\mathbf{n}$ instead of $\mathbf{n}_{\tau}$ if there is no confusion. Next, we define the unit normal vector on a face $e$. If $e \in \partial \Omega$, we define $\mathbf{n}_{e}$ as the unit normal vector pointing outside of $\Omega$. For a common face $e$ shared by two simplices, we let $\mathbf{n}_{e}$ be a fixed unit normal direction on $e$ common to the two simplices.

Now we are ready to define the function jumps. For a face $e$ shared by two simplices $\tau^{+}$and $\tau^{-}$, we use notations $\mathbf{v}^{+}$and $\mathbf{v}^{-}$to denote the values of a vector-valued $\mathbf{v}$ on $e$ taken from $\tau^{+}$and $\tau^{-}$, respectively. Then the jump of $\mathbf{v}$ over the face $e$ is defined as

$$
\left.[\mathbf{v}]\right|_{e}:=\left(\mathbf{n}_{\tau^{-}} \cdot \mathbf{n}_{e}\right) \mathbf{v}^{-}+\left(\mathbf{n}_{\tau^{+}} \cdot \mathbf{n}_{e}\right) \mathbf{v}^{+}
$$


and hence

$$
\left[\sigma \mathbf{n}_{e}\right]=\sigma^{+} \mathbf{n}_{\tau^{+}}+\sigma^{-} \mathbf{n}_{\tau^{-}} .
$$

For a face $e$ on the Dirichlet boundary $\partial \Omega_{D}$, we denote

$$
\left.[\mathbf{u}]\right|_{e}:=\mathbf{u}^{-}-\mathbf{u}_{b}
$$

where $\mathbf{u}^{-}$is the value of $\mathbf{u}$ on $e$ taken from the inside of $\Omega$. Moreover, for a face $e$ on the Neumann boundary $\partial \Omega_{N}$, we denote

$$
\left[\sigma \mathbf{n}_{e}\right]:=\sigma^{-} \mathbf{n}_{e}-\sigma_{b},
$$

where $\sigma^{-}$is the value of $\sigma$ on $e$ taken from the inside of $\Omega$.

\subsection{The DG method with staggered hybridization in subdomains}

Notice that for each subdomain $\Omega_{i}, \partial \Omega_{i}=\left(\partial \Omega_{i} \cap \partial \Omega_{D}\right) \cup\left(\partial \Omega_{i} \cap \partial \Omega_{N}\right) \cup\left(\bigcup_{(i, j) \in S} \Gamma_{i j}\right)$, where $S=\left\{(i, j): \Omega_{i}\right.$ and $\Omega_{j}$ have non-empty intersection $\}$. The initial problem on the entire domain is equivalent to the subdomain problems

$$
\begin{aligned}
\rho \frac{\partial \mathbf{u}}{\partial t}-\operatorname{div} \sigma=\mathbf{f}, & \text { in } \Omega_{i}, \\
\mathbf{A} \frac{\partial \sigma}{\partial t}-\varepsilon(\mathbf{u})=0, & \text { in } \Omega_{i} \\
\mathbf{u}=\mathbf{u}_{b}, & \text { on } \partial \Omega_{i} \bigcap \partial \Omega_{D}, \\
\sigma \mathbf{n}_{e}=\sigma_{b}, & \text { on } \partial \Omega_{i} \bigcap \partial \Omega_{N}, \\
\sigma \mathbf{n}_{e}=\sigma_{s}, & \text { on } \Gamma_{i j}, \quad(i, j) \in S,
\end{aligned}
$$

for a given vector-valued function $\sigma_{s}$ defined on $\Gamma$, which takes the value of $\sigma \mathbf{n}_{e}$ on each $\Gamma_{i j}$. In this subsection, we assume that the value of $\sigma_{s}$ is known and consider the DG method with staggered hybridization for the above problem in each subdomain $\Omega_{i}$. The computation of $\sigma_{s}$ and the combination of schemes on different subdomains will be discussed in the next subsection.

Let us denote $P^{k}(\tau)$ and $P^{k}(e)$ as the spaces of polynomials of degree at most $k$ defined on the simplex $\tau$ and face $e$, respectively. Now we introduce the following finite element spaces on $\Omega_{i}$ : the space of piecewise polynomials $U_{h, i}:=\left\{v:\left.v\right|_{\tau} \in P^{k}(\tau), \forall \tau \in \mathcal{T}_{i}\right\}$ and the space of $d \times d$ symmetric matrices $W_{h, i}:=\left\{\boldsymbol{\alpha}:\left.\boldsymbol{\alpha}\right|_{\tau} \in\left[P^{k}(\tau)\right]^{d \times d}, \boldsymbol{\alpha}=\boldsymbol{\alpha}^{T}, \forall \tau \in \mathcal{T}_{i}\right\}$. We approximate the solutions $\mathbf{u}$ and $\sigma$ on $\Omega_{i}$ with $\mathbf{u}_{h} \in\left[U_{h, i}\right]^{d}$ and $\sigma_{h} \in W_{h, i}$, respectively. By the definition, we know that the stress tensor $\sigma_{h}$ is always strongly symmetric. Note that by the definitions, $\mathbf{u}_{h}$ and $\sigma_{h}$ are just piecewise polynomials and thus can be discontinuous over the element faces. It is very easy to construct the basis of the finite element spaces.

Next, we define the spaces for Lagrange multipliers. Let us denote $\mathcal{F}_{u, i}^{D}=$ $\mathcal{F}_{u, i}^{0} \bigcup\left(\partial \Omega_{i} \cap \partial \Omega_{D}\right)$ and $\mathcal{F}_{p, i}^{N}=\mathcal{F}_{p, i} \bigcup\left(\partial \Omega_{i} \cap \partial \Omega_{N}\right)$. Moreover, we denote $\hat{W}_{h, i}:=\left\{\hat{\alpha}:\left.\hat{\alpha}\right|_{e} \in\right.$ 
$\left.P^{k}(e), \forall e \in \mathcal{F}_{u, i}^{D}\right\}$ and $\hat{U}_{h, i}:=\left\{\hat{v}:\left.\hat{v}\right|_{e} \in P^{k}(e), \forall e \in \mathcal{F}_{p, i}^{N}\right\}$. We approximate $\left.\mathbf{u}\right|_{\mathcal{F}_{p, i}^{N}}$ and $\left.\sigma \mathbf{n}_{e}\right|_{\mathcal{F}_{u, i}^{D}}$ with $\hat{\mathbf{u}}_{h} \in\left[\hat{U}_{h, i}\right]^{d}$ and $\hat{\sigma}_{h} \in\left[\hat{W}_{h, i}\right]^{d}$, respectively. To balance the degree of freedoms of the unknowns $\hat{\mathbf{u}}_{h}$ and $\hat{\sigma}_{h}$, we future impose the continuity of $\mathbf{u}_{h}$ on $\mathcal{F}_{u, i}^{D}$ and the continuity of $\sigma_{h} \mathbf{n}_{e}$ on $\mathcal{F}_{p, i}^{N}$ by the following conditions

$$
\begin{aligned}
& \int_{e}\left[\mathbf{u}_{h}\right] \cdot \hat{\boldsymbol{\alpha}}_{h}=0, \quad \forall \hat{\boldsymbol{\alpha}}_{h} \in\left[\hat{\boldsymbol{W}}_{h, i}\right]^{d}, \quad \forall e \in \mathcal{F}_{u, i}^{D} \\
& \int_{e}\left[\sigma_{h} \mathbf{n}_{e}\right] \cdot \hat{\mathbf{v}}_{h}=0, \quad \forall \hat{\mathbf{v}}_{h} \in\left[\hat{U}_{h, i}\right]^{d}, \quad \forall e \in \mathcal{F}_{p, i}^{N} .
\end{aligned}
$$

Notice that by the above conditions together with the notations (2.11) and (2.12), $\left.\mathbf{u}_{h}\right|_{\partial \Omega_{D}}$ and $\left.\sigma_{h} \mathbf{n}_{e}\right|_{\partial \Omega_{N}}$ computed from the inside of $\Omega$ are just the $L_{2}$ projections of the given boundary conditions.

To derive the DG method with staggered hybridization, we multiply Eq. (2.13) with a test function $\mathbf{v}_{h} \in\left[U_{h, i}\right]^{d}$ and multiply Eq. (2.14) with a test function $\boldsymbol{\alpha}_{h} \in W_{h, i}$, and then integrate these two equations on each $\tau \in \mathcal{T}_{i}$. By using integration by parts, the numerical solutions are required to satisfy

$$
\begin{array}{r}
\int_{\tau} \rho \frac{\partial \mathbf{u}_{h}}{\partial t} \cdot \mathbf{v}_{h}+\int_{\tau} \sigma_{h}: \nabla \mathbf{v}_{h}-\int_{\partial \tau \cap \mathcal{F}_{p, i}^{N}}\left(\sigma_{h} \mathbf{n}\right) \cdot \mathbf{v}_{h}-\int_{\partial \tau \cap \mathcal{F}_{u, i}^{D}}\left(\mathbf{n} \cdot \mathbf{n}_{e}\right) \hat{\boldsymbol{\sigma}}_{h} \cdot \boldsymbol{v}_{h} \\
-\sum_{(i, j) \in S} \int_{\partial \tau \cap \Gamma_{i j}}\left(\mathbf{n} \cdot \mathbf{n}_{e}\right) \sigma_{S} \cdot \boldsymbol{v}_{h}=\int_{\tau} \mathbf{f} \cdot \mathbf{v}_{h}, \\
\int_{\tau} A \frac{\partial \sigma_{h}}{\partial t}: \boldsymbol{\alpha}_{h}+\int_{\tau} \mathbf{u}_{h} \cdot \operatorname{div} \boldsymbol{\alpha}_{h}-\int_{\partial \tau \cap \mathcal{F}_{u, i}^{D}} \mathbf{u}_{h} \cdot\left(\boldsymbol{\alpha}_{h} \mathbf{n}\right)-\int_{\partial \tau \cap \mathcal{F}_{p, i}^{N}} \hat{\mathbf{u}}_{h} \cdot\left(\boldsymbol{\alpha}_{h} \mathbf{n}\right) \\
-\sum_{(i, j) \in S} \int_{\partial \tau \cap \Gamma_{i j}} \mathbf{u}_{h} \cdot\left(\boldsymbol{\alpha}_{h} \mathbf{n}\right)=0,
\end{array}
$$

for all test functions $\boldsymbol{v}_{h} \in\left[U_{h, i}\right]^{d}$ and $\boldsymbol{\alpha}_{h} \in W_{h, i}$, and for all $\tau \in \mathcal{T}_{i}$. Notice that the value of $\mathbf{u}_{h}$ on $\partial \tau \cap \Gamma_{i j}$ is taken from the inside of the current subdomain $\Omega_{i}$. Besides, due to the staggered continuity requirements in (2.18) and (2.19), all boundary terms in the above scheme are well defined.

\subsection{Mortar formulation}

To connect schemes for subdomain problems together, we need to define a proper mesh on the subdomain interfaces and the corresponding finite element space on it. Note that the triangulations among different subdomains can be non-matching. Hence, on each $\Gamma_{i j}$, there are two different meshes, which are respectively defined as the restrictions of $\mathcal{I} \mathcal{T}_{i}$ and $\mathcal{I} \mathcal{T}_{j}$ on $\Gamma_{i j}$. Among these two meshes, we choose the one with smaller mesh size as non-mortar mesh and denote it as $\mathcal{F}_{i j}$. The mesh on the other side is the mortar mesh and we denote it as $\mathcal{F}_{i j}^{m}$. Further more, we denote $\mathcal{F}_{\Gamma}=\bigcup_{(i, j) \in S} \mathcal{F}_{i j}$ and $\mathcal{F}_{\Gamma}^{m}=\bigcup_{(i, j) \in S} \mathcal{F}_{i j}^{m}$. For 
the non-mortar side, say $\Omega_{i}$, we introduce the space of Lagrange multipliers $M_{i j}=\left.U_{h, i}\right|_{\Gamma_{i j}}$, which consists of piecewise polynomial of degree up to $k$ defined on $\Gamma_{i j}$ with respect to the mesh $\mathcal{F}_{i j}$. In addition, we let $M_{h}=\prod_{(i, j) \in S}\left[M_{i j}\right]^{d}$, which is a finite element space defined on $\mathcal{F}_{\Gamma}$.

We determine additional unknown $\sigma_{s, h} \in M_{h}$ on $\Gamma$ to approximate the boundary value $\sigma_{s}$ used in the local subdomain problems. Moreover, we need to enforce continuity of the solutions of the above subdomain problems across subdomain interfaces by

$$
\int_{\Gamma_{i j}}\left(\left.\boldsymbol{u}_{h}\right|_{\Omega_{i}}-\left.\boldsymbol{u}_{h}\right|_{\Omega_{j}}\right) \cdot \boldsymbol{\mu}=0, \quad \forall(i, j) \in S,
$$

for all test function $\mu \in M_{h}$. By introducing the extra unknown and additional conditions, we can then combine schemes in different subdomains together. To simplify the notation, we define $\mathcal{F}_{u}^{D}=\left(\bigcup_{i=1}^{N} \mathcal{F}_{u, i}^{D}\right) \cup \mathcal{F}_{\Gamma}$ and $\mathcal{F}_{p}^{N}=\bigcup_{i=1}^{N} \mathcal{F}_{p, i}^{N}$. Also, we let $U_{h}=\prod_{i=1}^{N}\left[U_{h, i}\right]^{d}, W_{h}=$ $\prod_{i=1}^{N} W_{h, i}, \hat{U}_{h}=\prod_{i=1}^{N}\left[\hat{U}_{h, i}\right]^{d}$, and $\tilde{W}_{h}=\prod_{i=1}^{N}\left[\hat{W}_{h, i}\right]^{d} \otimes M_{h}$. Moreover, we define $\tilde{\sigma}_{h} \in \tilde{W}_{h}$ as

$$
\begin{array}{cl}
\left.\tilde{\sigma}_{h}\right|_{e}=\hat{\sigma}_{h}, & \text { for } e \in \mathcal{F}_{u, i}^{D}, \\
\left.\tilde{\sigma}_{h}\right|_{e}=\sigma_{h, s}, & \text { for } e \in \mathcal{F}_{\Gamma},
\end{array}
$$

which is a numerical approximation to $\sigma \mathbf{n}_{e}$ on $\mathcal{F}_{u}^{D}$. By using the above notations, we know that the DG method in the entire domain seeks for numerical solutions $\mathbf{u}_{h}=\mathbf{u}_{h}(t) \in$ $U_{h}, \sigma_{h}=\sigma_{h}(t) \in W_{h}, \hat{\mathbf{u}}_{h}=\hat{\mathbf{u}}_{h}(t) \in \hat{U}_{h}$, and $\tilde{\sigma}_{h}=\tilde{\sigma}_{h}(t) \in \tilde{W}_{h}$ to approximate $\left.\mathbf{u}\right|_{\Omega},\left.\sigma\right|_{\Omega},\left.\mathbf{u}\right|_{\mathcal{F}_{p}^{N}}$, and $\left.\sigma \mathbf{n}_{e}\right|_{\mathcal{F}_{u}^{D}}$, respectively.

Next, we illustrate that our scheme can be solved locally. For each $e \in \mathcal{F}_{u, i}^{0}$ we define $\mathcal{R}(e)$ to be the union of simplices in $\mathcal{T}_{i}$ sharing the face $e$. For a face $e \in \mathcal{F}_{u, i} \cap \partial \Omega$, we let $\mathcal{R}(e)$ be the simplex in $\mathcal{T}_{i}$ having the face $e$. To simplify the code, we assume that each face in $\mathcal{F}_{i j}^{m}$ is a union of several faces in $\mathcal{F}_{i j}$. In this case, for each $e \in \mathcal{F}_{i j}^{m}$, we define $\mathcal{R}(e)$ as the union of the simplices on both sides sharing the face $e$ or part of $e$. We denote $\mathcal{F}_{u}^{0}=\left(\bigcup_{i=1}^{N} \mathcal{F}_{u, i}^{0}\right) \cup \mathcal{F}_{\Gamma}^{m}, \mathcal{F}_{p}=\bigcup_{i=1}^{N} \mathcal{F}_{p, i}$ and $\mathcal{F}_{u}=\bigcup_{i=1}^{N}\left(\mathcal{F}_{u, i} \backslash\left(\mathcal{F}_{u, i} \cap \Gamma\right)\right) \cup \mathcal{F}_{\Gamma}^{m}$, then we easily see that $\left\{\mathcal{R}(e), e \in \mathcal{F}_{u}\right\}$ is a partition of $\Omega$. Also, $\{\mathcal{S}(v), v \in \mathcal{N}\}$ is another partition of $\Omega$, where $\mathcal{N}=\bigcup_{i=1}^{N} \mathcal{N}_{i}$.

In order to compute our scheme locally, we sum (2.20) on each $\mathcal{R}(e), e \in \mathcal{F}_{u}$ and (2.21) on each $\mathcal{S}(v), v \in \mathcal{N}$. By using the above notations, we summarise the mortar DG method with staggered hybridization as: find the unique solutions $\mathbf{u}_{h} \in U_{h}, \sigma_{h} \in W_{h}, \hat{\mathbf{u}}_{h} \in \hat{U}_{h}$, and $\tilde{\boldsymbol{\sigma}}_{h} \in \tilde{W}_{h}$ such that, for all test functions $\mathbf{v}_{h} \in U_{h}, \boldsymbol{\alpha}_{h} \in W_{h}, \hat{\mathbf{v}}_{h} \in \hat{U}_{h}$, and $\tilde{\boldsymbol{\alpha}}_{h} \in \tilde{W}_{h}$ we have

$$
\begin{aligned}
\int_{\mathcal{R}(e)} \rho \frac{\partial \mathbf{u}_{h}}{\partial t} \cdot \mathbf{v}_{h}+B_{e}\left(\sigma_{h}, \mathbf{v}_{h}\right) & -\sum_{e \cap \mathcal{F}_{u}^{0}} D_{e}^{*}\left(\tilde{\boldsymbol{\sigma}}_{h}, \mathbf{v}_{h}\right)-\int_{e \cap \partial \Omega_{D}} \tilde{\sigma}_{h} \cdot \mathbf{v}_{h}=\int_{\mathcal{R}(e)} \mathbf{f} \cdot \mathbf{v}_{h}, \quad \forall e \in \mathcal{F}_{u}, \\
\int_{\mathcal{S}(v)} A \frac{\partial \sigma_{h}}{\partial t}: \boldsymbol{\alpha}_{h}-B_{v}^{*}\left(\mathbf{u}_{h}, \boldsymbol{\alpha}_{h}\right) & +\sum_{e \in \mathcal{S}(v) \cap \mathcal{F}_{p}} D_{e}\left(\hat{\mathbf{u}}_{h}, \boldsymbol{\alpha}_{h}\right) \\
& -\int_{\mathcal{S}(v) \cap \partial \Omega_{N}} \hat{\mathbf{u}}_{h} \cdot\left(\boldsymbol{\alpha}_{h} \mathbf{n}_{e}\right)=0, \quad \forall v \in \mathcal{N}
\end{aligned}
$$




$$
\begin{aligned}
& D_{e}^{*}\left(\tilde{\boldsymbol{\alpha}}_{h}, \mathbf{u}_{h}\right)=0, \quad \forall e \in \mathcal{F}_{u}^{D}, \\
& D_{e}\left(\hat{\mathbf{v}}_{h}, \sigma_{h}\right)=0, \quad \forall e \in \mathcal{F}_{p}^{N},
\end{aligned}
$$

where

$$
\begin{aligned}
& B_{e}(\boldsymbol{\alpha}, \mathbf{v}):=\int_{\mathcal{R}(e)} \boldsymbol{\alpha}: \nabla \mathbf{v}-\int_{\partial \mathcal{R}(e) \cap \mathcal{F}_{p}^{N}}(\boldsymbol{\alpha} \mathbf{n}) \cdot \mathbf{v}, \\
& B_{v}^{*}(\mathbf{v}, \boldsymbol{\alpha}):=-\int_{\mathcal{S}(v)} \mathbf{v} \cdot \operatorname{div} \boldsymbol{\alpha}+\int_{\partial \mathcal{S}(v) \cap \mathcal{F}_{u}^{D}} \mathbf{v} \cdot(\boldsymbol{\alpha} \mathbf{n}), \\
& D_{e}(\hat{\mathbf{v}}, \boldsymbol{\alpha}):=-\int_{e} \hat{\mathbf{v}} \cdot\left[\boldsymbol{\alpha} \mathbf{n}_{e}\right], \\
& D_{e}^{*}(\tilde{\boldsymbol{\alpha}}, \mathbf{v}):=\int_{e} \tilde{\boldsymbol{\alpha}} \cdot[\mathbf{v}] .
\end{aligned}
$$

The choices of the mesh and solutions spaces lead to the energy conservation property of our scheme. In particular, we take $\mathbf{v}_{h}=\mathbf{u}_{h}$ in (2.25) and take $\boldsymbol{\alpha}_{h}=\sigma_{h}$ in (2.26), we have

$$
\begin{array}{r}
\int_{\mathcal{R}(e)} \rho \frac{\partial \mathbf{u}_{h}}{\partial t} \cdot \mathbf{u}_{h}+B_{e}\left(\sigma_{h}, \mathbf{u}_{h}\right)-\int_{\partial \Omega_{D}} \tilde{\sigma}_{h} \cdot \mathbf{u}_{\mathbf{b}}=\int_{\mathcal{R}(e)} \mathbf{f} \cdot \mathbf{u}_{h}, \quad \forall e \in \mathcal{F}_{u}, \\
\int_{\mathcal{S}(v)} A \frac{\partial \sigma_{h}}{\partial t}: \sigma_{h}-B_{v}^{*}\left(\mathbf{u}_{h}, \sigma_{h}\right)-\int_{\partial \Omega_{N}} \hat{\mathbf{u}}_{h} \cdot \sigma_{b}=0, \quad \forall v \in \mathcal{N},
\end{array}
$$

where we applied Eqs. (2.27) and (2.28). Summing Eqs. (2.33) and (2.34) over all $e \in \mathcal{F}_{u}$ and $v \in \mathcal{N}$, and using the mortar condition (2.22), we have

$$
\frac{1}{2} \frac{d}{d t}\left(\int_{\Omega} \rho\left|\mathbf{u}_{h}\right|^{2}+\int_{\Omega} A \sigma_{h}: \sigma_{h}\right)=\int_{\Omega} \mathbf{f} \cdot \mathbf{u}_{h}+\int_{\partial \Omega_{D}} \tilde{\sigma}_{h} \cdot \mathbf{u}_{\mathbf{b}}+\int_{\partial \Omega_{N}} \hat{\mathbf{u}}_{h} \cdot \sigma_{b},
$$

which gives the required energy conservation property.

\subsection{Leap-frog in time}

We use the standard leap-frog scheme, which is explicit and energy conserving, for the time discretization. We compute $\boldsymbol{u}_{h}$ and $\tilde{\boldsymbol{\sigma}}_{h}$ at times $\left\{t_{h}\right\}$, and compute $\sigma_{h}$ and $\hat{\boldsymbol{u}}_{h}$ at times $\left\{t_{n+\frac{1}{2}}\right\}$.

Given $\boldsymbol{u}_{h}^{n}, \tilde{\sigma}_{h}^{n}$ and $\sigma_{h}^{n+\frac{1}{2}}$, we can compute $\boldsymbol{u}_{h}^{n+1}$ and $\tilde{\boldsymbol{\sigma}}_{h}^{n+1}$ locally on each $\mathcal{R}(e), e \in \mathcal{F}_{u}$ by solving $D_{e}^{*}\left(\tilde{\boldsymbol{\alpha}}_{h}, \boldsymbol{u}_{h}^{n+1}\right)=0\left(\right.$ on $\left.e \cap \mathcal{F}_{u}^{D}\right)$ and

$$
\begin{aligned}
& \int_{\mathcal{R}(e)} \rho \frac{\boldsymbol{u}_{h}^{n+1}-\boldsymbol{u}_{h}^{n}}{\Delta t} \cdot \boldsymbol{v}_{h}-\frac{1}{2} \sum_{e \cap \mathcal{F}_{u}^{0}} D_{e}^{*}\left(\tilde{\boldsymbol{\sigma}}_{h}^{n+1}, \boldsymbol{v}_{h}\right)-\frac{1}{2} \int_{e \cap \partial \Omega_{D}} \tilde{\boldsymbol{\sigma}}_{h}^{n+1} \cdot \boldsymbol{v}_{h} \\
= & \int_{\mathcal{R}(e)} f\left(\boldsymbol{x}, t_{n+\frac{1}{2}}\right) \cdot \boldsymbol{v}_{h}-B_{e}\left(\boldsymbol{\sigma}_{h}^{n+\frac{1}{2}}, \boldsymbol{v}_{h}\right)+\frac{1}{2} \sum_{e \cap \mathcal{F}_{u}^{0}} D_{e}^{*}\left(\tilde{\boldsymbol{\sigma}}_{h}^{n}, \boldsymbol{v}_{h}\right)+\frac{1}{2} \int_{e \cap \partial \Omega_{D}} \tilde{\boldsymbol{\sigma}}_{h}^{n} \cdot \boldsymbol{v}_{h},
\end{aligned}
$$


for all test functions $\boldsymbol{v}_{h} \in\left[U_{h}\right]^{d}$ and $\tilde{\boldsymbol{\alpha}}_{h} \in\left[\tilde{W}_{h}\right]^{d}$. Given $\boldsymbol{\sigma}_{h}^{n+\frac{1}{2}}, \hat{\boldsymbol{u}}_{h}^{n+\frac{1}{2}}$ and $\boldsymbol{u}_{h}^{n+1}$, we can compute $\sigma_{h}^{n+\frac{3}{2}}$ and $\hat{\boldsymbol{u}}_{h}^{n+\frac{3}{2}}$ locally on each $\mathcal{S}(v), v \in \mathcal{N}$ by solving $D_{e}\left(\hat{\boldsymbol{v}}_{h}, \sigma_{h}^{n+\frac{3}{2}}\right)=0\left(\right.$ on $\left.e \in \mathcal{S}(v) \cap \mathcal{F}_{p}^{N}\right)$ and

$$
\begin{aligned}
& \int_{\mathcal{S}(v)} A \frac{\sigma_{h}^{n+\frac{3}{2}}-\sigma_{h}^{n+\frac{1}{2}}}{\Delta t}: \boldsymbol{\alpha}_{h}+\frac{1}{2} \sum_{e \in \mathcal{S}(v) \cap \mathcal{F}_{p}} D_{e}\left(\hat{\boldsymbol{u}}_{h}^{n+\frac{3}{2}}, \boldsymbol{\alpha}_{h}\right)-\frac{1}{2} \int_{\mathcal{S}(v) \cap \partial \Omega_{N}} \hat{\mathbf{u}}_{h}^{n+\frac{3}{2}} \cdot\left(\boldsymbol{\alpha}_{h} \mathbf{n}_{e}\right) \\
= & B_{v}^{*}\left(\boldsymbol{u}_{h}^{n+1}, \boldsymbol{\alpha}_{h}\right)-\frac{1}{2} \sum_{e \in \mathcal{S}(v) \cap \mathcal{F}_{p}} D_{e}\left(\hat{\boldsymbol{u}}_{h}^{n+\frac{1}{2}}, \boldsymbol{\alpha}_{h}\right)+\frac{1}{2} \int_{\mathcal{S}(v) \cap \partial \Omega_{N}} \hat{\mathbf{u}}_{h}^{n+\frac{1}{2}} \cdot\left(\boldsymbol{\alpha}_{h} \mathbf{n}_{e}\right),
\end{aligned}
$$

for all test functions $\boldsymbol{\alpha}_{h} \in W_{h}$ and $\hat{\boldsymbol{v}}_{h} \in\left[\hat{U}_{h}\right]^{d}$. We remark that the above systems are defined locally on $\mathcal{R}(e)$ and $\mathcal{S}(v)$ respectively, and can therefore be solved very efficiently.

\section{Numerical results}

\section{Example 1. Convergence test}

In this example, we will test the convergence rate of our mortar formulation. We take $\rho=10, \mu=2.704$ and $\lambda=264.992$. The corresponding Poisson's ratio is 0.495 , which is relatively high. The exact solutions are taken as $\mathbf{u}(x, y, t)=[\sin (y+t), \cos (x-t)]^{T}, \sigma_{11}=$ $\sigma_{22}=0$ and $\sigma_{12}(x, y, t)=\sigma_{21}(x, y, t)=\mu(\sin (y+t)-\cos (x-t))$, and hence the source term is $\mathbf{f}(x, y, t)=[(\rho-\mu) \cos (y+t),(\rho-\mu) \sin (x-t)]^{T}$. The computational domain is $\Omega=[0,2 \pi]^{2}$. The upper boundary $y=2 \pi$ is set to be the Neumann boundary and the other three boundaries are the Dirichlet boundaries.

We divide the computational domain into two parts with the interface locating at $y=\frac{4 \pi}{3}$, and use a fine mesh for the upper part and a coarse mesh for the lower part. For simplicity, we divide the lower part into $N \times N$ uniform rectangles and further subdivide each rectangle into two triangles. We use these triangles as our initial mesh. For the upper part, we use unstructured triangulation. The edge length of the triangles on the boundary is set to be $\frac{2 \pi}{3 N}$. A sample mesh with $N=4$ is shown in Fig. 2. In our simulations, we use a fine enough time step size so that the dominant error comes from the spatial discretization. For $k=1$, we take $\Delta=0.01 h$, while for $k=2$, we take $\Delta t=0.02 h^{1.5}$, where $h=\frac{2 \pi}{3 N}$. We present the convergence history with weighted $L^{2}$ norms $\|u\|_{\rho}^{2}=\int_{\Omega} \rho u^{2} d x$ and $\|\sigma\|_{\mathbf{A}}^{2}=\int_{\Omega} \mathbf{A} \sigma: \sigma d x$ at the time $T=0.01$ in Table 1 . We can observe optimal convergence rate. We also tried the Poisson's ratio 0.251 and obtained similar results.

\section{Example 2. Lamb's problem}

We test our method by the Lamb's problem, which is a classical test case for the implementation of free surface boundary condition. The problem setting is as follows. The density, the velocity for the P-wave and S-wave are 1500, 520, 300 respectively and the corresponding Poissons ratio is 0.251 . The computation domain is $[0,280] \times[-140,0]$ and 


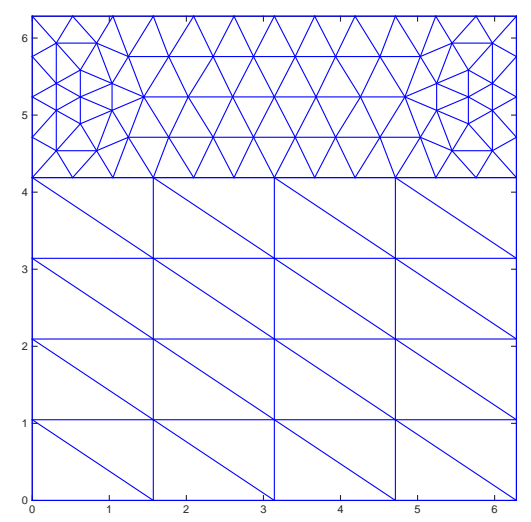

Figure 2: A sample mesh with $N=4$ for Example 1 .

Table 1: Convergence history of Example 1.

\begin{tabular}{||c|c|c|c|c|c|c||}
\hline$N$ & $\left\|\left(\mathbf{u}_{h}\right)_{1}-u_{1}\right\|_{\rho}$ & Order & $\left\|\left(\mathbf{u}_{h}\right)_{2}-u_{2}\right\|_{\rho}$ & Order & $\left\|\sigma_{h}-\sigma\right\|_{\mathbf{A}}$ & Order \\
\hline & \multicolumn{7}{|c||}{$k=1$} \\
\hline 4 & $3.66 \mathrm{e}-02$ & - & $8.61 \mathrm{e}-02$ & - & $5.04 \mathrm{e}-02$ & - \\
8 & $9.47 \mathrm{e}-03$ & 1.95 & $2.18 \mathrm{e}-02$ & 1.98 & $1.31 \mathrm{e}-02$ & 1.94 \\
16 & $2.46 \mathrm{e}-03$ & 1.95 & $5.56 \mathrm{e}-03$ & 1.97 & $3.33 \mathrm{e}-03$ & 1.98 \\
32 & $6.60 \mathrm{e}-04$ & 1.90 & $1.42 \mathrm{e}-03$ & 1.97 & $8.17 \mathrm{e}-04$ & 2.03 \\
64 & $1.77 \mathrm{e}-04$ & 1.90 & $3.53 \mathrm{e}-04$ & 2.01 & $2.08 \mathrm{e}-04$ & 1.97 \\
\hline \multicolumn{7}{||c|}{$k=2$} \\
\hline 4 & $3.25 \mathrm{e}-03$ & - & $9.95 \mathrm{e}-03$ & - & $5.67 \mathrm{e}-03$ & - \\
8 & $4.47 \mathrm{e}-04$ & 2.86 & $1.27 \mathrm{e}-03$ & 2.97 & $7.21 \mathrm{e}-04$ & 2.98 \\
16 & $5.90 \mathrm{e}-05$ & 2.92 & $1.61 \mathrm{e}-04$ & 2.98 & $8.91 \mathrm{e}-05$ & 3.02 \\
32 & $7.65 \mathrm{e}-06$ & 2.95 & $2.01 \mathrm{e}-05$ & 3.00 & $1.13 \mathrm{e}-05$ & 2.97 \\
64 & $1.09 \mathrm{e}-06$ & 2.80 & $2.61 \mathrm{e}-06$ & 2.95 & $1.56 \mathrm{e}-06$ & 2.87 \\
\hline
\end{tabular}

the free surface is set at depth $z=0$. A vertical point force is applied to the free surface at $(x, z)=(140,0)$. The force is given by a Ricker wavelet defined by the first derivative of

$$
w(t)=2 \pi f\left(t-t_{0}\right) e^{-\pi^{2} f^{2}\left(t-t_{0}\right)^{2}},
$$

where the frequency $f=50$ and $t_{0}=0.024$.

It is well known that the Rayleigh wave travels along the free surface and decays exponentially in the vertical direction. To capture the detailed structure near the free surface, a dense mesh would be used. On the other hand, the structure of the solution is not complicated in the area far away from the free surface and the costly dense mesh is 

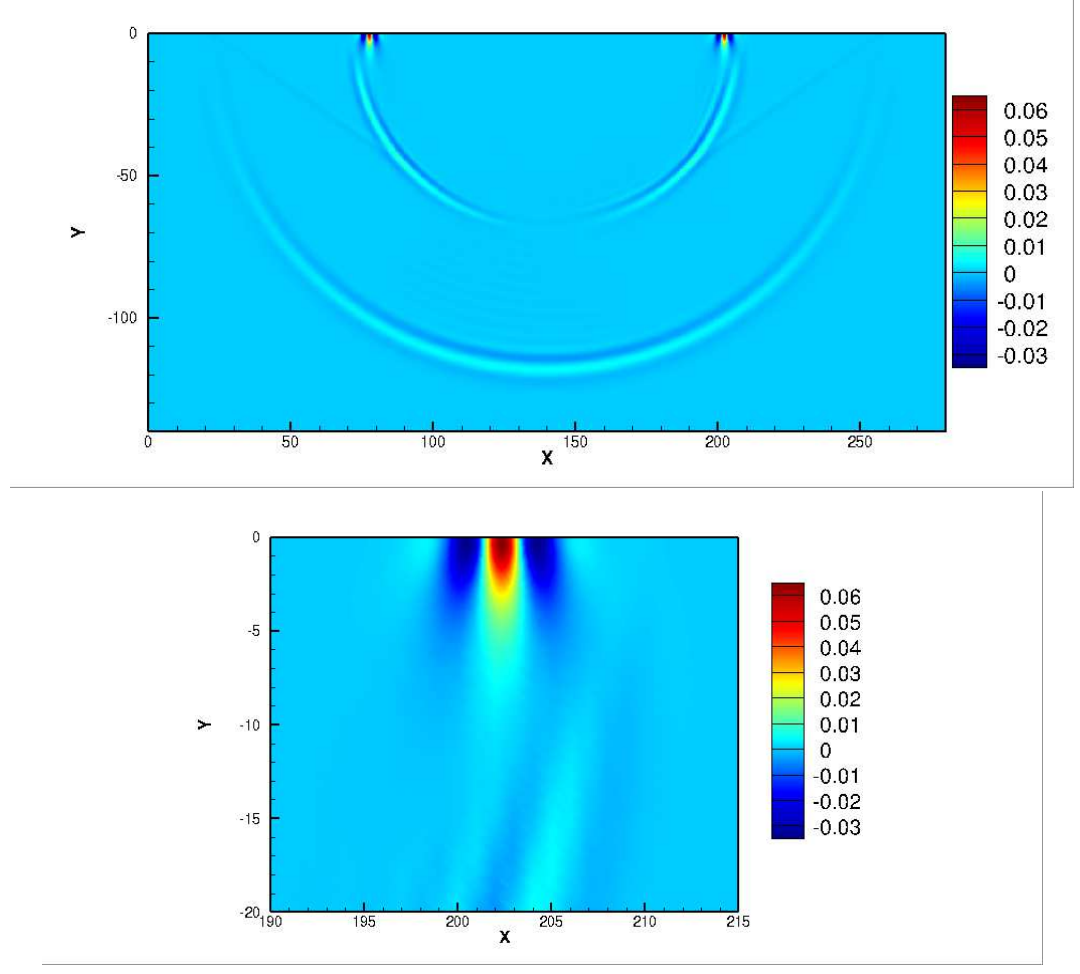

Figure 3: Lamb's Problem. The velocity $u_{2}$ at time $t=0.25 \mathrm{~s}$.

unnecessary. Hence, we use the mortar formulation to save the total computational cost. We divide the computational domain into two parts with the interface locating at the depth $z=-6$. For the upper part which is a thin layer near the free surface, we use a fine unstructured triangulation. The edge length of the triangles on the boundary is set to be $h=\frac{1}{3}$. For the rest of the domain, we use a coarse mesh. Here we adopt a structured mesh to further reduce the computational cost. We first divide the lower part into $280 \times 134$ uniform squares and further divide them into triangles. Hence, the edge length of the squares is $3 h$.

The plot of $u_{2}$ at time $t=0.25 \mathrm{~s}$ and its zoom-in picture are shown in Fig. 3. From this figure, we can observe the faster P-wave and the slower S-wave as well as the Rayleigh wave. Also, we see that the Rayleigh wave is traveling along the free surface, and its speed is slightly smaller than that of the S-wave. To show the accuracy of the mortar formulation, we also compute a reference solution by using the SDG method on a globally fine mesh with the edge length as $h$. Fig. 4 shows the sample meshes with $h=\frac{8}{3}$. In the real computation, we take $h=\frac{1}{3}$ as mentioned before. In Fig. 5, we present the comparison at four different observation points $(160,-5),(180,-5),(200,-5)$, and $(220,-5)$. From these comparisons, we see clearly that the mortar formulation gives a very accurate solution. 


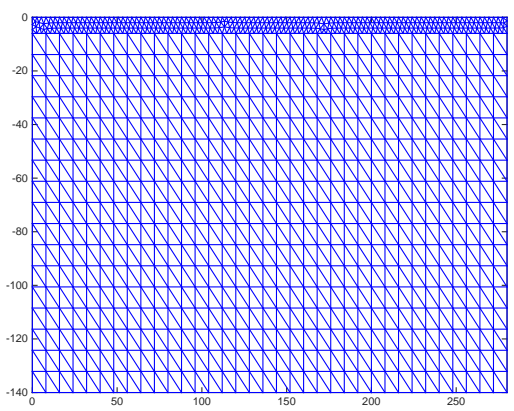

(a) mortar mesh

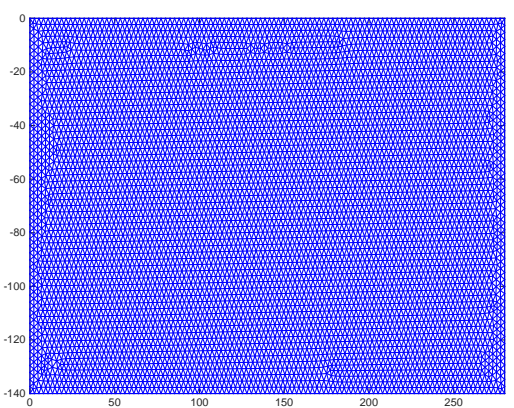

(b) globally fine mesh

Figure 4: Samples of different meshes.

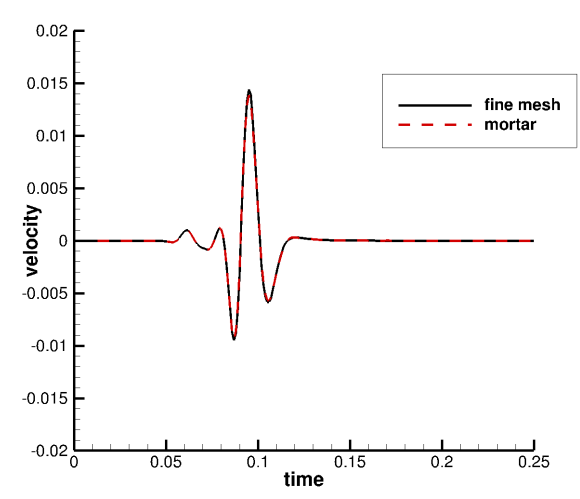

(a) $(x, z)=(160,-5)$

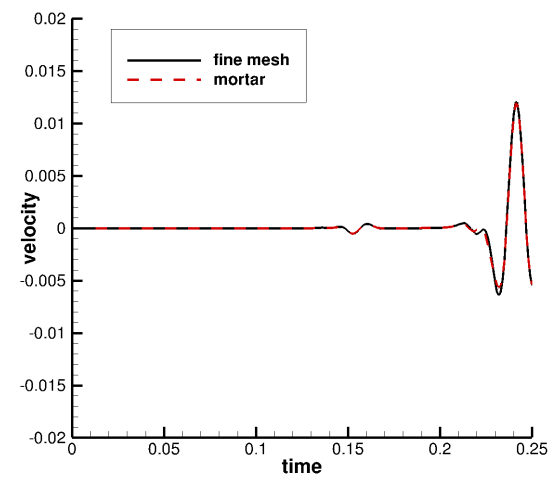

(c) $(x, z)=(200,-5)$

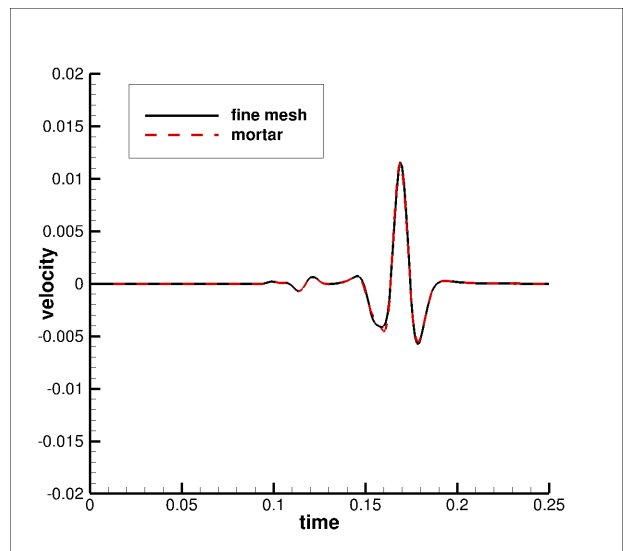

(b) $(x, z)=(180,-5)$

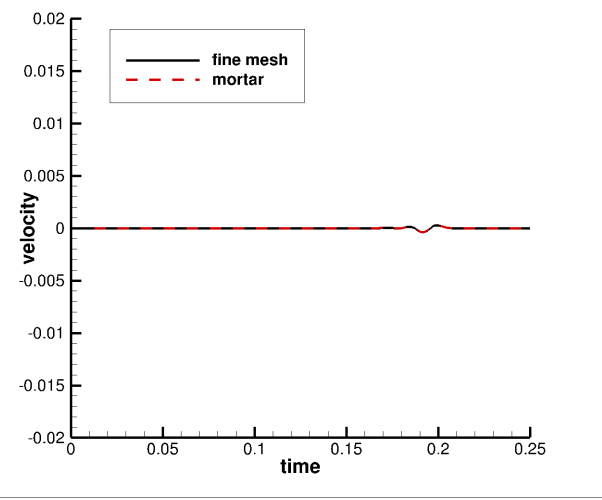

(d) $(x, z)=(220,-5)$

Figure 5: Comparison of our solution (mortar formulation) to a reference solution (fine mesh) at various observation points. 


\section{Example 3. Heterogeneous material}

In this example, we will stimulate Rayleigh waves in a heterogeneous material with vertical variations in velocity structure on scales smaller than a wavelength. The domain of interest is still $[0,280] \times[-140,0]$ and the setting of the vertical point force is the same as in the last example. The density is $1500 \mathrm{kgm}^{-3}$. The P- and S-wave velocities $v_{p}$ and $v_{s}$ are described as follows:

$$
\left(v_{p}, v_{s}\right)(x, z)= \begin{cases}(520,300), & \text { if } 0 \leqslant z \leqslant-1 \text { or }-2 \leqslant z \leqslant-3 \\ (400,155), & \text { otherwise. }\end{cases}
$$

We use the same mesh as in the last example. The snapshot of the solution $u_{2}$ at the simulation time $T=0.341 \mathrm{~s}$ is shown in Fig. 6 . From this figure, we see clearly that the dispersive behavior of the Rayleigh wave for a vertically varying velocity model is accurately captured.

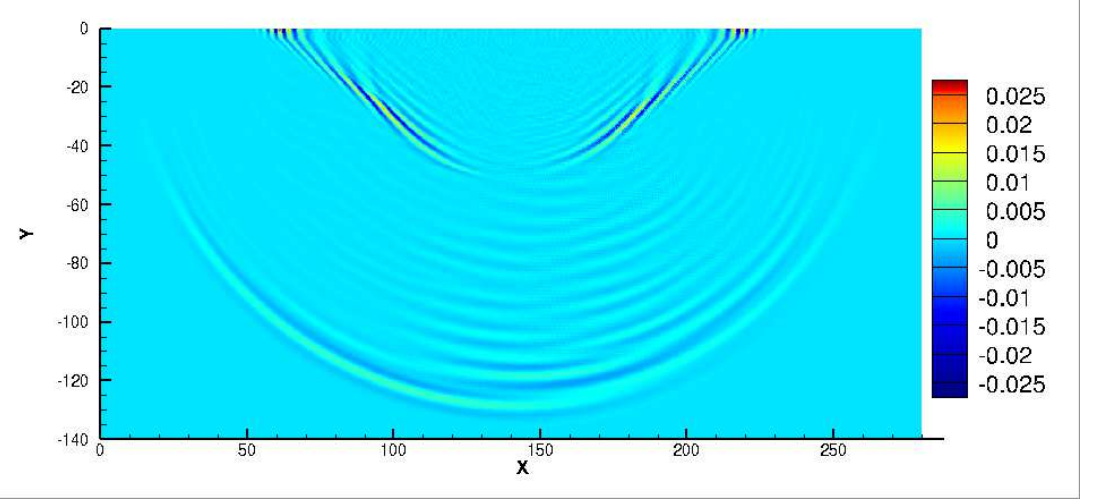

Figure 6: Heterogeneous material. The velocity $u_{2}$ at the time $t=0.341$.

\section{Example 4. Curved surface topography}

In the last example, we demonstrate the ability of our method to simulate Rayleigh waves in a domain with surface topography. The physical parameters are the same as in the second example. The computational domain is shown in Fig. 7. In the middle of the top boundary, there is a convex shaped region followed by a concave shaped region. The point source is vertically excited at $(140,8)$, which is at the top part of the convex region of the free surface. We divide the computational domain into two parts with the interface locating at the depth $z=-10$. As in the previous examples, we take an unstructured mesh with the cell length $h$ for the upper part and take a coarse structured mesh with the cell length $3 h$ for the lower part. Fig. 7 shows a sample mesh with $h=\frac{2}{3}$. In real computation, we take $h=\frac{1}{3}$. Fig. 8 shows the plots of $u_{2}$ at different times. We can observe the P-wave, $\mathrm{S}$-wave and the Rayleigh wave as well as the influence of the curved shaped surface. 


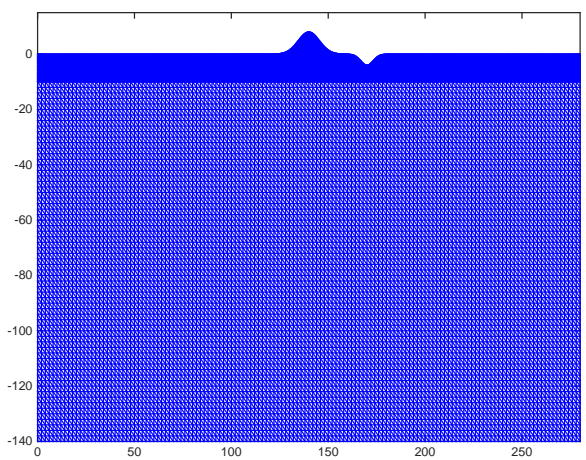

Figure 7: Sample mesh on the domain with curved shaped surface.

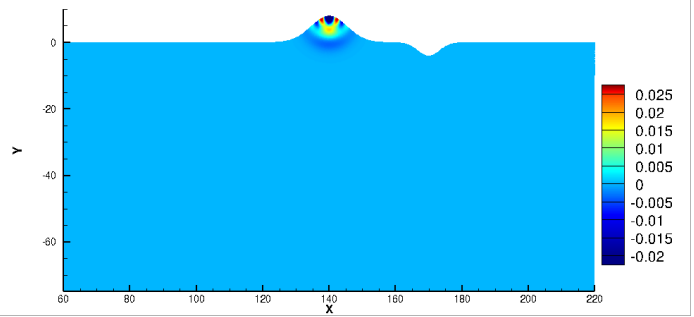

(a) $t=0.0320$

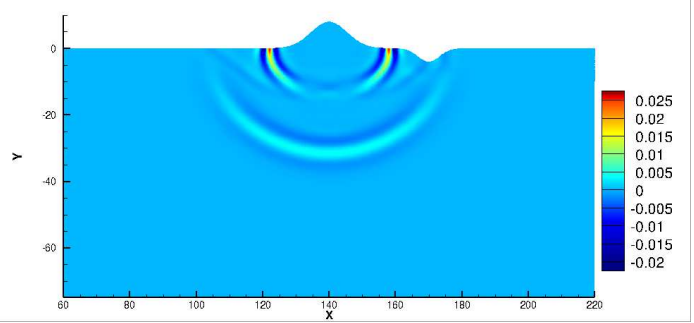

(c) $t=0.0984$

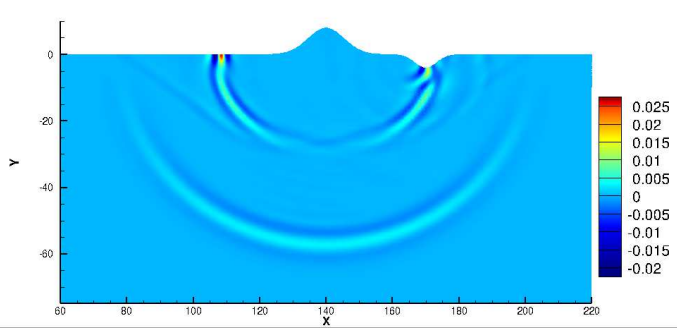

(e) $t=0.148$

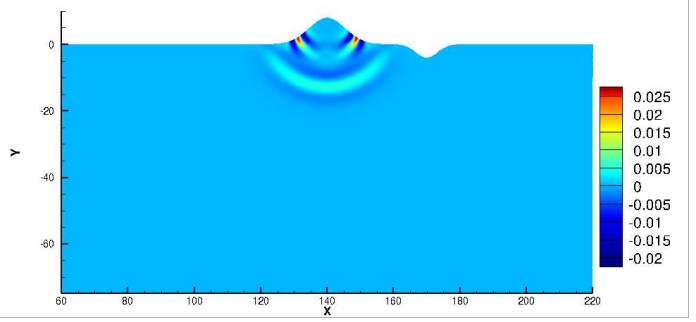

(b) $t=0.0624$

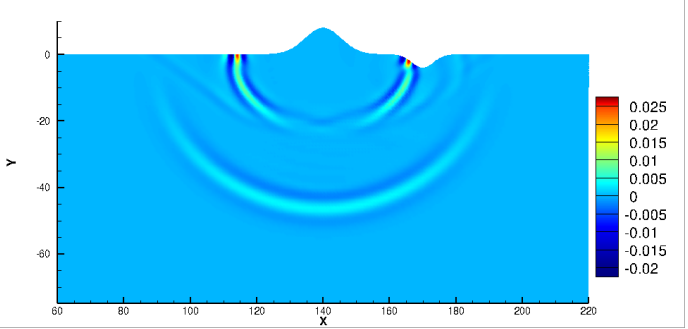

(d) $t=0.1272$

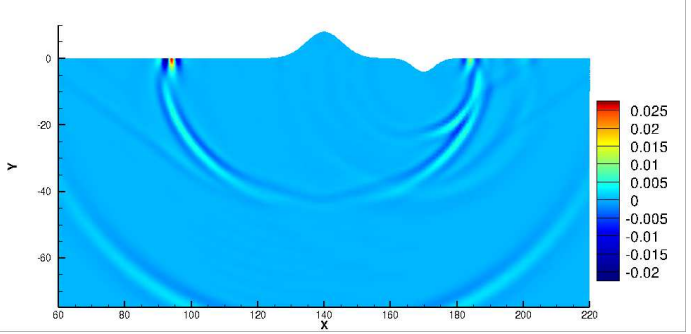

(f) $t=0.2$

Figure 8: Domain with curved boundary. The velocity $u_{2}$ at different times. 


\section{Conclusion}

In this paper, we present a mortar discontinuous Galerkin method for the simulation of elastic waves and Rayleigh waves. The proposed method is motivated by the staggered grid finite difference schemes. The key features of the proposed methods are the use of discontinuous Galerkin method coupled with staggered hybridization and mortar formulation. Our scheme uses fine mesh near the surface wave and coarse mesh in most part of the computational domain. This results in a significant reduction in computational cost. In addition, our scheme gives high order accuracy, energy conservation and preservation of symmetry of stress tensor. We present realistic numerical examples to demonstrate the performance of the proposed method. In our forthcoming paper, we will present the convergence analysis for our method.

\section{Acknowledgments}

Jie Du's work is supported by the National Natural Science Foundation of China under grant number NSFC 11801302. Eric Chung's work is partially supported by the Hong Kong RGC General Research Fund (Project numbers 14304719 and 14302018) and the CUHK Faculty of Science Direct Grant 2018-19.

\section{References}

[1] P.F. Antonietti, I. Mazzieri, A. Quarteroni, and F. Rapetti. Non-conforming high order approximations of the elastodynamics equation. Computer Methods in Applied Mechanics and Engineering, 209212:212 - 238, 2012.

[2] Hiu Ning Chan, Eric T. Chung, and Gary Cohen. Stability and dispersion analysis of the staggered discontinuous Galerkin method for wave propagation. International Journal of $\mathrm{Nu}$ merical Analysis \& Modeling, 10(1), 2013.

[3] Siu Wun Cheung, Eric Chung, Hyea Hyun Kim, and Yue Qian. Staggered discontinuous Galerkin methods for the incompressible Navier-Stokes equations. Journal of Computational Physics, 302:251-266, 2015.

[4] Ching-Shan Chou, Chi-Wang Shu, and Yulong Xing. Optimal energy conserving local discontinuous Galerkin methods for second-order wave equation in heterogeneous media. Journal of Computational Physics, 272:88-107, 2014.

[5] Eric Chung, Bernardo Cockburn, and Guosheng Fu. The staggered DG method is the limit of a hybridizable DG method. SIAM Journal on Numerical Analysis, 52(2):915-932, 2014.

[6] Eric T. Chung, Jie Du, and Chi Yeung Lam. Discontinuous galerkin methods with staggered hybridization for linear elastodynamics. Computers \& Mathematics with Applications, 74(6):1198-1214, 2017.

[7] Eric T. Chung and Björn Engquist. Optimal discontinuous Galerkin methods for wave propagation. SIAM Journal on Numerical Analysis, 44(5):2131-2158, 2006.

[8] Eric T. Chung and Björn Engquist. Optimal discontinuous Galerkin methods for the acoustic wave equation in higher dimensions. SIAM Journal on Numerical Analysis, 47(5):3820-3848, 2009. 
[9] Eric T. Chung, Chi Yeung Lam, and Jianliang Qian. A staggered discontinuous Galerkin method for the simulation of seismic waves with surface topography. Geophysics, 80(4):T119$\mathrm{T} 135,2015$.

[10] Bernardo Cockburn, Jayadeep Gopalakrishnan, and Raytcho Lazarov. Unified hybridization of discontinuous Galerkin, mixed, and continuous Galerkin methods for second order elliptic problems. SIAM Journal on Numerical Analysis, 47(2):1319-1365, 2009.

[11] Bernardo Cockburn and Vincent Quenneville-Bélair. Uniform-in-time superconvergence of the HDG methods for the acoustic wave equation. Mathematics of Computation, 83(285):65-85, 2014.

[12] Bernardo Cockburn and Ke Shi. Superconvergent HDG methods for linear elasticity with weakly symmetric stresses. IMA Journal of Numerical Analysis, 33(3):747-770, 2013.

[13] Appelö Daniel and Thomas Hagstrom. A new discontinuous Galerkin formulation for wave equations in second-order form. SIAM Journal on Numerical Analysis, 53(6):2705-2726, 2015.

[14] Jonás D. De Basabe, Mrinal K. Sen, and Mary F. Wheeler. The interior penalty discontinuous Galerkin method for elastic wave propagation: grid dispersion. Geophysical Journal International, 175(1):83-93, 2008.

[15] Sarah Delcourte, Loula Fezoui, and Nathalie Glinsky-Olivier. A high-order discontinuous Galerkin method for the seismic wave propagation. In ESAIM: Proceedings, volume 27, pages 70-89. EDP Sciences, 2009.

[16] Leszek Demkowicz and JT Oden. Application of hp-adaptive BE/FE methods to elastic scattering. Computer methods in applied mechanics and engineering, 133(3):287-317, 1996.

[17] Jie Du, Eric T. Chung, Ming Fai Lam, and Xiao-Ping Wang. Discontinuous Galerkin method with staggered hybridization for a class of nonlinear Stokes equations. Journal of Scientific Computing, 76(3):1547-1577, 2018.

[18] V Etienne, E Chaljub, J Virieux, and N Glinsky. An hp-adaptive discontinuous Galerkin finite-element method for 3-D elastic wave modelling. Geophysical Journal International, 183(2):941-962, 2010.

[19] Martin Käser and Michael Dumbser. An arbitrary high-order discontinuous Galerkin method for elastic waves on unstructured meshesi. the two-dimensional isotropic case with external source terms. Geophysical Journal International, 166(2):855-877, 2006.

[20] Jeonghun J. Lee and Hyea Hyun Kim. Analysis of a staggered discontinuous Galerkin method for linear elasticity. Journal of Scientific Computing, pages 1-25, 2015.

[21] Alan R Levander. Fourth-order finite-difference P-SV seismograms. Geophysics, 53(11):14251436, 1988.

[22] Hailiang Liu and Jue Yan. The direct discontinuous Galerkin (DDG) method for diffusion with interface corrections. Communications in Computational Physics, 8(3):541, 2010.

[23] Raul Madariaga. Dynamics of an expanding circular fault. Bulletin of the Seismological Society of America, 66(3):639-666, 1976.

[24] PJ Matuszyk, LF Demkowicz, and C Torres-Verdín. Solution of coupled acoustic-elastic wave propagation problems with anelastic attenuation using automatic hp-adaptivity. Computer Methods in Applied Mechanics and Engineering, 213:299-313, 2012.

[25] N.C. Nguyen, J. Peraire, and B. Cockburn. High-order implicit hybridizable discontinuous Galerkin methods for acoustics and elastodynamics. Journal of Computational Physics, 230(10):3695-3718, 2011.

[26] Weifeng Qiu, Jiguang Shen, and Ke Shi. An HDG method for linear elasticity with strong symmetric stresses. arXiv preprint arXiv:1312.1407, 2013.

[27] Béatrice Rivière, Simon Shaw, Mary F. Wheeler, and J.R. Whiteman. Discontinuous Galerkin 
finite element methods for linear elasticity and quasistatic linear viscoelasticity. Numerische Mathematik, 95(2):347-376, 2003.

[28] Jason P Sheldon, Scott T. Miller, and Jonathan S. Pitt. An improved formulation for hybridizable discontinuous Galerkin fluid-structure interaction modeling with reduced computational expense. Communications in Computational Physics, 24(SAND-2018-0096J), 2018.

[29] Jean Virieux. P-SV wave propagation in heterogeneous media: Velocity-stress finitedifference method. Geophysics, 51(4):889-901, 1986.

[30] Lucas C. Wilcox, Georg Stadler, Carsten Burstedde, and Omar Ghattas. A high-order discontinuous Galerkin method for wave propagation through coupled elastic-acoustic media. Journal of Computational Physics, 229(24):9373-9396, 2010.

[31] Lina Zhao, Eric Chung, and Ming Fai Lam. A new staggered DG method for the Brinkman problem robust in the Darcy and Stokes limits. arXiv preprint arXiv:1911.08759, 2019. 